\title{
Review \\ Tumour-stroma interactions \\ Reciprocal regulation of extracellular matrix proteins and ovarian steroid activity in the mammary gland
}

\author{
Sandra Z Haslam and Terry L Woodward \\ Department of Physiology, Michigan State University, Michigan, USA
}

Correspondence: Sandra Z Haslam, 108 Giltner Hall, Department of Physiology, Michigan State University, East Lansing , MI 48824, USA.

Tel: +1 5173556475 Ext.1232; fax: +1 517355 5125; e-mail: shaslam@msu.edu

Received: 17 December 2000

Breast Cancer Res 2001, 3:365-372

Revisions requested: 31 January 2001

Revisions received: 30 April 2001

Accepted: 30 June 2001

Published: 2 August 2001

(C) 2001 BioMed Central Ltd

(Print ISSN 1465-5411; Online ISSN 1465-542X)

\begin{abstract}
Despite the critical importance of ovarian steroids in the treatment of breast cancer, little is known about the acquisition or loss of estrogen and progesterone responsiveness in either the normal or neoplastic mammary gland. This review focuses on the interactions among mammary stroma-derived extracellular matrix (ECM) proteins, integrins and ovarian hormone-dependent proliferation in normal and neoplastic mammary cells both in vivo and in vitro. In vitro studies show that fibronectin is required for progesterone-induced proliferation of normal mammary epithelial cells and that specific ECM proteins also regulate interactions between growth factors and ovarian hormones. Studies with human breast cancer cell lines have shown that laminin inhibits estrogen-induced proliferation and estrogenresponse-element-mediated transcription in vitro and also inhibits estrogen-induced proliferation in vivo. Reciprocally, ovarian steroids regulate the expression of ECM proteins and their cellular receptors, integrins, during mammary gland development in vivo. The fibronectin-specific integrin, $\alpha_{5} \beta_{1}$ is regulated by ovarian steroids and its expression is positively correlated with developmental stages of peak proliferation. These studies suggest that the coordinated regulation of ovarian hormone responsiveness and $\mathrm{ECM}$ /integrin expression may be critical to normal mammary gland development and breast cancer growth and progression.
\end{abstract}

Keywords: breast cancer, estrogen, extracellular matrix, integrins, progesterone

\section{Introduction}

Postnatal mammary gland development and function are highly dependent upon the actions of the ovarian hormones, estrogen and progesterone [1]. In estrogen receptor knockout (ERKO) mice only a rudimentary mammary gland is present at birth and no further development occurs postnatally [2]. To determine whether estrogen receptor $(E R)$ is required in stromal tissue, epithelial tissue or both for normal mammary gland development, Cunha et al. [3] have surgically transplanted wild-type $(E R+/+)$ or ERKO (ER-/-) epithelial tissue with wild-type or ERKO stromal tissue under the kidney capsule of athymic nude mice. These authors reported that ER+/+ stroma was required for normal mammary ductal morphogenesis, but $\mathrm{ER}+/+$ epithelium was not. These results corroborate in vitro studies that have demonstrated that estrogen-dependent mammary epithelial proliferation and/or ductal morphogenesis is mediated through the paracrine action of

$\mathrm{BM}=$ basement membrane; $\mathrm{ECM}=$ extracellular matrix; $\mathrm{EGF}=$ epidermal growth factor; $\mathrm{ER}=$ estrogen receptor; ERE = estrogen response element; ERKO = estrogen receptor knockout; IGF-I = insulin-like growth factor-I; IGFBP = insulin-like growth factor binding protein; PR = progesterone receptor. 
estrogen-induced, stroma-derived factors. Stromal cells appear to influence epithelial cell behavior by the secretion of growth factors and/or by altering the composition of the extracellular matrix (ECM) in which epithelial cells reside [4-6]. Previously we have reviewed the regulation and action of autocrine and paracrine growth factors (including the epidermal growth factor, insulin-like growth factor, hepatocyte growth factor and fibroblast growth factor families) involved in ovarian steroid-mediated epithelial-stromal interactions [4]. A recent report has also demonstrated that keratinocyte growth factor mRNA and protein are increased by estrogen in mammary stroma, although it is not clear what effect keratinocyte growth factor has on mammary gland development or differentiation [7]. In the past few years, several studies have revealed information about basement membrane (BM) ECM proteins and their cellular receptors: they regulate cell proliferation, differentiation and apoptosis; they are secreted and/or regulated by complex epithelial-stromal interactions; they are regulated by ovarian steroids; and they regulate ovarian steroid action. Hansen and Bissell [8] have recently reviewed experimental models that have been developed to study the effects of ECM, steroids and growth factors on breast cancer. This review will focus on recent findings, by us and other investigators, about ECM composition and integrin expression in vitro and in vivo in relation to the proliferative responses of normal and neoplastic mammary epithelial cells to estrogen and progestins.

Although animal models, including the ERKO mice study described above, have been invaluable in determining a requirement for epithelial-stromal interactions in ovarian steroid-mediated mammary morphogenesis, cell culture models have been useful to elucidate the underlying mechanisms of cell-cell interactions and the cell types involved. Many previous culture studies, however, that were performed to address these questions in the mammary gland were carried out in the presence of serum or impure supplements (e.g. matrigel, fetuin, pituitary extracts). Serum and these supplements contain ECM proteins, growth factors and other biologically relevant components that have impeded our understanding of the specific mechanisms by which stromal cells contribute to the acquisition and loss of ovarian hormone responsiveness. We carried out the in vitro studies, described in this review, under serum-free, defined culture conditions. This has allowed us to address these issues more precisely.

\section{Influence of ECM proteins on steroid-induced proliferation in vitro}

ECM proteins work in concert with systemic hormones and autocrine/paracrine growth factors to regulate proliferation, differentiation and apoptosis. Mammary epithelial cells and myoepithelial cells are separated from stromal cells by a BM composed of ECM proteins. The origin of the BM was once considered to be synthesized exclu- sively by epithelial cells, but now researchers have determined, in several epithelial tissues, that the BM requires epithelial stromal interactions and that often most resident ECM proteins in the BM are synthesized by mesenchymal or stromal cells. Mammary adipocytes have been shown to produce laminin and heparan sulfate proteoglycans; fibroblasts produce collagen I, fibronectin and tenascin in vivo $[9,10]$. Keely et al. [10] have presented compelling evidence based upon in situ hybridization and immunocytochemical analyses, that mammary stromal cells are primarily responsible for the expression of collagen I, collagen IV and laminin in the mammary gland. Perhaps the most meticulous examination of the cellular origin of BM synthesis and deposition has occurred in the gut. Simon-Assman et al. have found that BM components of the gut are of dual origin, with heparan sulfate proteoglycans being produced primarily by epithelial cells, while collagen and laminin were produced mainly by stromal cells [11]. Using novel mouse/chick tissue recombinants and analyzing ECM expression with species-specific antibodies, they have further determined that alpha- 2 and alpha-4 laminin chains are produced exclusively by stromal cells. The laminin alpha- 5 chain, however, is expressed by both epithelial and stromal cells in a developmentally regulated pattern. These studies demonstrate a complex interaction between epithelial and stromal cells in the formation of the BM [12]. These researchers have also demonstrated that the formation of a BM requires contact between epithelial and mesenchymal cells. Similar studies in the mammary gland are lacking. Taken together, however, these studies strongly suggest that epithelial-stromal interactions are critical for the formation, and perhaps regulation, of the epithelial BM in the mammary gland. ECM proteins, therefore, like growth factors, can function as a stromal-derived paracrine factor that may influence epithelial behavior.

To determine if ECM proteins affect ovarian steroid action on mammary epithelium, we have investigated the interactions between ECM proteins (collagens I and IV, laminin, fibronectin and tenascin) and estrogen and progestin in serum-free primary cell culture of mouse mammary epithelial cells [5]. These studies demonstrated that ovarian steroid-induced proliferation of epithelial cells only occurred when epithelial cells were cultured on certain ECM proteins. Epithelial cells derived from adult, virgin mice proliferated in response to the progestin R5020, only on fibronectin and collagen IV (Fig. 1). The response to R5020 on these matrices could not be explained by differences in ER or progesterone receptor (PR) levels, or by differences in cell attachment. Estrogen does not induce proliferation of mammary epithelial cells in vitro, unless epithelial cells are co-cultured with stromal cells [13]. In monoculture, however, epithelial cells also exhibited a proliferative response to estrogen, albeit inconsistently, on fibronectin, suggesting that the estrogen-induced 


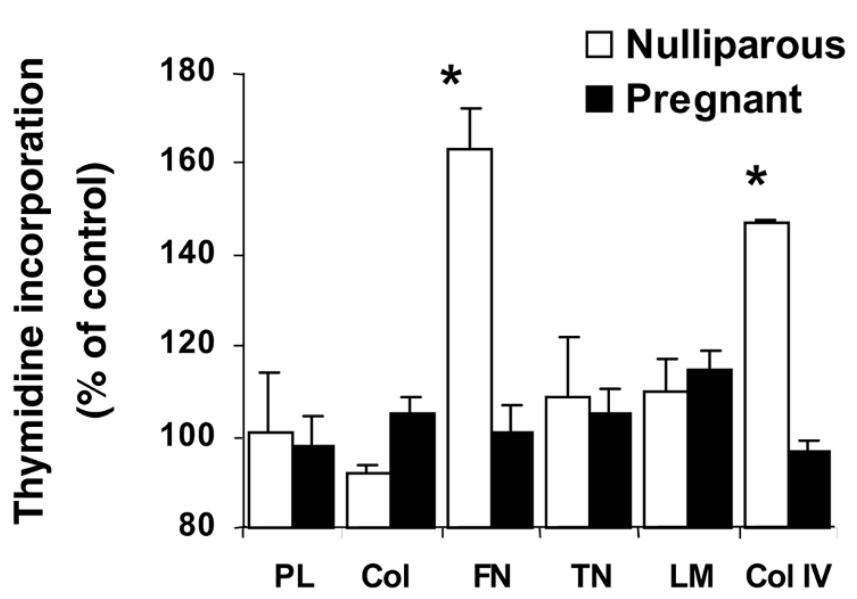

The effect of progestin (R5020) and extracellular matrix proteins on mammary epithelial DNA synthesis. Epithelial cells derived from nulliparous or pregnant mice were plated on poly-L-lysine $(\mathrm{PL})$, collagen I (Col I), fibronectin (FN), tenascin (TN), laminin (LN), or collagen IV (Col IV) in serum-free medium. At 24 hour post-plating, the media was changed to either the control media or a media containing $\mathrm{R} 5020(20 \mathrm{nM}) .{ }^{3} \mathrm{H}$-thymidine incorporation into DNA was determined 48 hour later. The percent increase was obtained by dividing counts per $\mathrm{min} / \mathrm{mg}$ DNA of cells cultured in the presence of R5020 by counts per $\mathrm{min} / \mathrm{mg}$ DNA of cells cultured in the absence of R5020. ${ }^{*} P<0.05$ that R5020 stimulated proliferation of nulliparous derived cells on FN and Col IV. (Adapted from [3] with permission from Endocrinology).

response may require several stromal signals, including ECM. Epithelial cells from pregnant mice were not responsive to estrogen or R5020, on any matrix. Thus, responsiveness to ovarian hormones was first and foremost dependent upon the inherent state of mammary gland differentiation, but within the framework of mammary gland differentiation, ECM molecules can modulate hormonal responsiveness.

\section{Interactions among growth factors, ovarian hormones and ECM proteins in vitro}

There is considerable evidence to indicate that the proliferative effects of estrogen are mediated indirectly by locally produced stroma-derived growth factors [4]. There is also evidence that growth factors can synergize with estrogen or can activate ER in the absence of ligand [14]. To address this issue we have investigated the effects of specific ECM proteins on the proliferative responses to epidermal growth factor (EGF) and insulin-like growth factor-I (IGF-I), and their interactions with estrogen and $\mathrm{R} 5020$ [6]. All ECM proteins tested in these experiments (collagens I and IV, fibronectin and laminin), promoted highly synergistic proliferation between EGF and IGF-I (Fig. 2a) This synergism was accomplished through several mechanisms, including increased expression of growth factor receptors. EGF receptor levels were significantly increased on collagen I and fibronectin, whereas
Figure 2
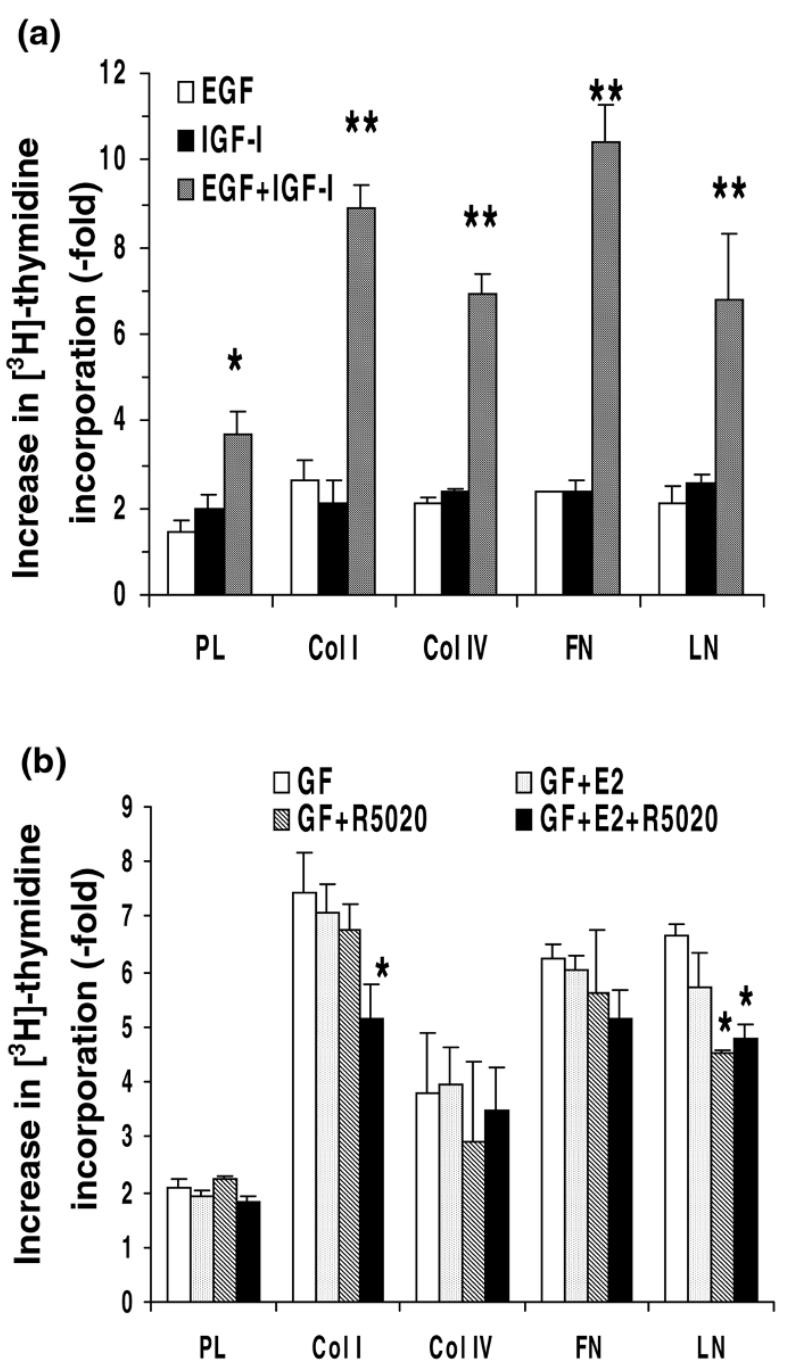

Epithelial cell proliferation on different extracellular matrix proteins induced by epidermal growth factor plus insulin-like growth factor-l. Epithelial cells derived from nulliparous mice were plated as described in Fig. 1a. (a) At 24 hour post-plating, the media was changed to control (no growth factors), or a media containing EGF $(50 \mathrm{ng} / \mathrm{ml}), \mathrm{IGF}-\mathrm{I}$ (300 ng/ml) or EGF+IGF-I (50 ng/ml + $300 \mathrm{ng} / \mathrm{ml})$. ${ }^{3} \mathrm{H}$-thymidine incorporation into DNA was determined 24 hour later. ${ }^{*} P<0.01$ that on poly-L-lysine, proliferation in EGF+IGF-I treated group is greater than in EGF- or IGF-I-treated groups. ${ }^{* *} P<0.01$ that on all ECM proteins, proliferation in EGF+IGF-I is greater than in EGF- or IGF-I-treated groups on ECM proteins and poly-L-lysine. (b) Epithelial cells were plated on indicated ECM proteins in medium without growth factors or hormones (control), with growth factors (GF: EGF $25 \mathrm{ng} / \mathrm{ml}+$ IGF-I $100 \mathrm{ng} / \mathrm{ml})$, with or without estrogen (E2, $10 \mathrm{nM})$ and/or R5020 (23 $\mathrm{nM}) .{ }^{*} P=0.05$ that values obtained with EGF + IGF-I + R5020 on LN and with EGF + IGF-I + E + R5020 on Col I and LN are significantly lower than EGF + IGF-I. (Adapted from [4] with permission from Endocrinology). Col, collagen; E2, estrogen; ECM, extracellular matrix; EGF, epidermal growth factor; FN, fibronectin; GF, growth factors; IGF-I, insulin-like growth factor-I; LN, laminin; PL, poly-L-lysine.

IGF-I receptor levels were increased on all of the ECM proteins tested, with the greatest increase observed on 
laminin. The synergism between the two growth factors was also mediated by ECM effects on IGF-I binding protein (IGFBP) levels. IGFBP-2 and -3 were increased in cells cultured with IGF-I alone, but decreased in the presence of EGF + IGF-I. The cells cultured on fibronectin, however, did not show this trend. Overall, levels of IGFBPs were lower in cells cultured on fibronectin. The decrease in IGFBPs that occurred with EGF + IGF-I or in the presence of fibronectin may facilitate increased IGF-I activity.

No additive or synergistic proliferative effect was observed with EGF, IGF-I or EGF + IGF-I and estrogen or R5020. In fact, the opposite was observed; estrogen + R5020 decreased proliferation in an ECM-dependent manner when added with EGF + IGF-I to cells cultured on collagen I or laminin (Fig. 2b). In vivo studies investigating the expression of and response to IGF-I have shown that IGF-I is important for ductal elongation in the immature gland, but not during pregnancy [15]. In contrast, PR levels are low and progestins are ineffective in stimulating proliferation at this stage of development, but play an important role in alveolar morphogenesis during pregnancy [16]. Thus, the expression of IGF-I and IGF-I receptors and PR and progestin responsiveness are inversely related and appear to be important in two distinct stages of proliferation: ductal elongation versus alveolar morphogenesis. In this context, our findings that R5020 + estrogen reduce the proliferative response to EGF + IGF-I in vitro also suggest that progestins may have an inhibitory effect on IGF-l-induced proliferation in vivo. The observations from these studies demonstrate that various stroma-derived ECM proteins regulate specific aspects of the response and are necessary for EGF-IGF-I synergism. Furthermore, this synergism may be reduced by progestins.

\section{Developmental and hormonal regulation of fibronectin and its $\alpha_{5} \beta_{1}$ integrin, in vivo}

Since specific ECM proteins influence mammary epithelial cell proliferative response to hormones and growth factors in vitro, it was of interest to investigate their roles in vivo. Toward this end, we have analyzed the in vivo spatial and temporal concentrations of fibronectin, collagens I and IV and laminin during postnatal development, and the effects of ovariectomy and estrogen and progesterone treatment on ECM and integrin expression [17].

We observed that the levels of the ECM protein fibronectin and its classic receptor, the $\alpha_{5} \beta_{1}$ integrin, are developmentally and hormonally regulated in the normal mouse mammary gland. Western blot analysis revealed that fibronectin levels increased 3 -fold between the prepubertal state and sexual maturity, remaining high during pregnancy and lactation. Fibronectin increased only in intact glands in association with the epithelium. In contrast to fibronectin, the levels of collagens I and IV and laminin did not exhibit
Figure 3

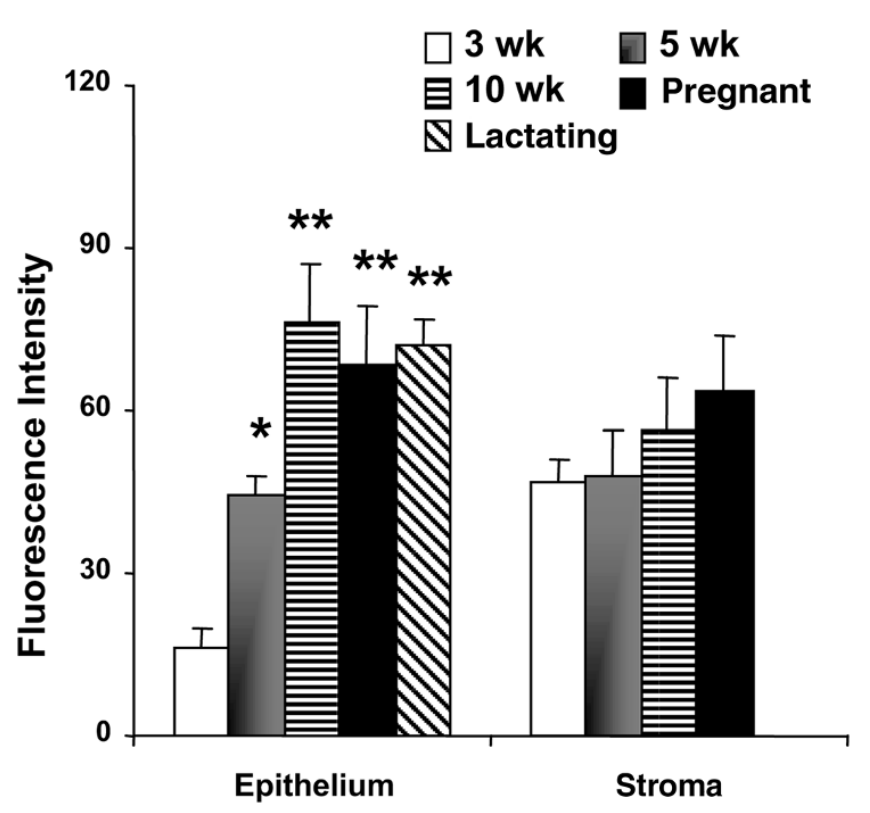

Fibronectin expression in normal mammary gland as a function of age and developmental state. Frozen sections of mammary glands from immature, pubertal, sexually mature (3-, 5-, 10-week-old, respectively), or pregnant and lactating mice were immunostained with antibody to fibronectin. Fluorescence staining intensity (measured as average pixel brightness) was quantitated for epithelial and stromal cells as previously described [20]. ${ }^{\star} P<0.05$ that 10 -week-old, pregnant and lactating epithelial staining intensity is greater than that of 3- or 5week-old mice.

major changes during development. Fibronectin levels decreased by $70 \%$ after ovariectomy and were increased 1.5- and 2-fold by treatment with estrogen or estrogen + progesterone, respectively (Fig. 3).

During development, $\alpha_{5} \beta_{1}$ integrin levels increase from puberty to early pregnancy and decrease at late pregnancy and lactation (Fig. 4a). Analysis of hormonal regulation of the $\alpha_{5} \beta_{1}$ integrin in the adult, virgin mammary gland revealed that the levels of the $\alpha_{5} \beta_{1}$ integrin was significantly down-regulated by ovariectomy and rapidly (by 24 hours after treatment) up-regulated after estrogen + progesterone treatment in both mammary epithelial and myoepithelial cells (Fig. 4b). The subcellular localization of the $\alpha_{5} \beta_{1}$ integrin was also developmentally and hormonally regulated. During periods of active proliferation, such as puberty and early pregnancy, and in ovariectomized adult mice following estrogen + progesterone treatment, the $\alpha_{5} \beta_{1}$ integrin was preferentially localized to the basal surface of the cell. Hormone-dependent basal localization of the $\alpha_{5} \beta_{1}$ integrin is suggestive of binding to adjacent BM proteins and is consistent with integrin activation and signaling during estrogen-mediated or progesterone-mediated mitogenesis. High basal localization of $\alpha_{5} \beta_{1}$ was reduced during late pregnancy and lactation, 

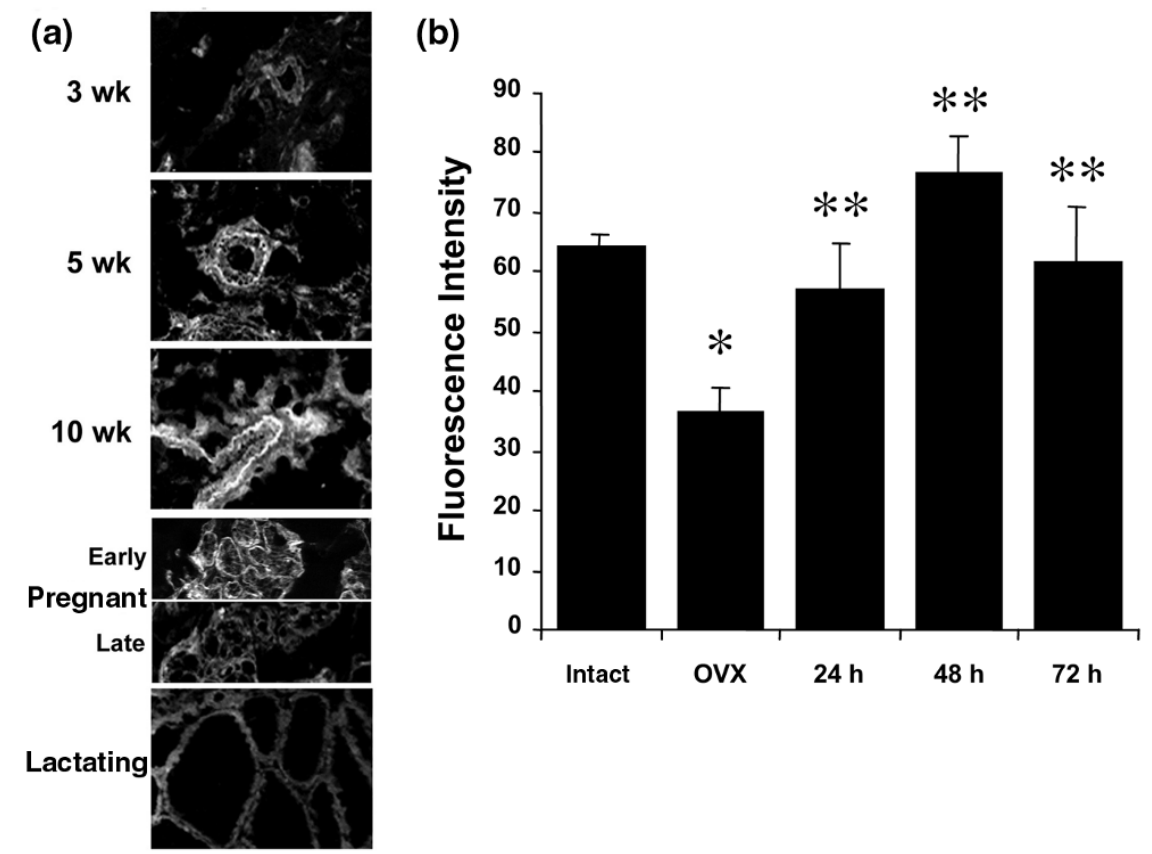

Cellular localization and ovarian steroid regulation of $\alpha_{5} \beta_{1}$ integrin in mouse mammary glands during development. (a) Frozen sections of mammary glands from immature, pubertal, sexually mature (3-, 5-, 10-week-old, respectively), early pregnant (9-12 days), late pregnant (19-21 days) and lactating (7-10 days) mice were immunostained with antibody to the $\alpha_{5} \beta_{1}$ integrin. Note that staining is seen around ducts at 5 and 10 weeks of age and around alveoli in early pregnant mice. No staining was seen at 3 weeks of age or at late pregnancy and lactation. Mag. 100X.

(b) Mammary glands were removed from 10-week-old mice that were ovary intact (intact), ovariectomized for 1 week (OVX), or OVX given a single i.p. injection containing $17 \beta$-estradiol $(1 \mu \mathrm{g})+$ progesterone $(1 \mathrm{mg})(E+P)$ and sacrificed 24,48 or 72 hour later. Frozen sections were stained with antibody to $\alpha_{5} \beta_{1}$ integrin and fluorescence staining intensity (measured as average pixel brightness) was quantitated [20]. ${ }^{\star} P<0.05$ that OVX $\alpha_{5} \beta_{1}$ integrin staining intensity is lower than intact. ${ }^{*} P<0.05$ that at 24,48 , and $72 \mathrm{hr}$ post $\mathrm{E}+\mathrm{P}$ injection, $\alpha_{5} \beta_{1}$ integrin staining intensity is greater than OVX.

developmental stages characterized by low proliferation. This leads us to hypothesize that fibronectin and the $\alpha_{5} \beta_{1}$ integrin play an important role in estrogen-dependent and estrogen + progesterone-dependent regulation of epithelial cell proliferation. Because $\alpha_{5} \beta_{1}$ integrin levels were more closely correlated with proliferation than fibronectin levels, and because the $\alpha_{5} \beta_{1}$ integrin was more rapidly regulated by ovarian steroids than fibronectin, it is likely that regulation of integrin expression represents a more dynamic mechanism for controlling cellular proliferation and morphogenesis than the synthesis and degradation of ECM proteins.

\section{ECM effects on estrogen action in human breast cancer cells in vitro and in vivo}

Deciphering the complex interactions involved in growth factor-dependent and ovarian-steroid-dependent proliferation in the normal mammary gland has specific relevance to understanding the alterations in the growth control that occur in breast cancer. Loss of estrogen responsiveness in breast cancer cells is a major problem in the treatment of this disease. Long-term treatment of estrogen-responsive breast cancer with antiestrogen has not been effec- tive because tumors eventually become antiestrogen resistant [18]. Despite the critical importance to breast cancer treatment, the mechanism(s) leading to loss of estrogen responsiveness and antiestrogen resistance is poorly understood.

In breast cancer, the stromal cells surrounding tumors have increased or altered the expression of many ECM proteins that change with breast cancer progression [19-21]. However, little is known about how specific ECM proteins might affect estrogen action in breast cancer cells. To address this question, we have studied the effects of various ECM proteins on estrogen-induced proliferation in two ER positive human breast cancer cell lines (MCF-7, T47D) in serum-free cell culture [22]. We found that one ECM protein, laminin, specifically inhibited estrogen-induced proliferation and estrogen-induced PR induction in MCF-7 and T47D cells (Fig. 5a,b). The effect of laminin was specific for estrogen since it did not inhibit the proliferative response to the growth factors, EGF or IGF-I. Our results indicate that the inhibition of estrogen action by laminin is a postreceptor, postbinding event because ER concentration and ER binding are not altered by 
(a) $\square$ Control Control
Estrogen

- Estrogen

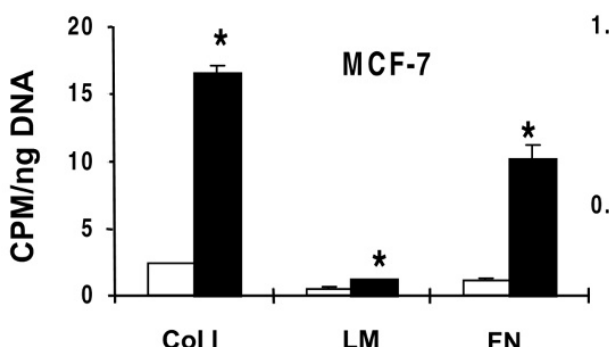

Coll

LM

FN

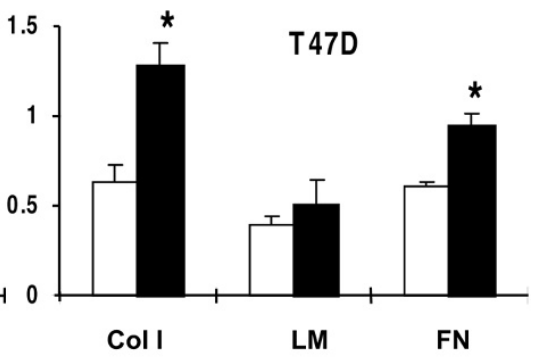

(b)

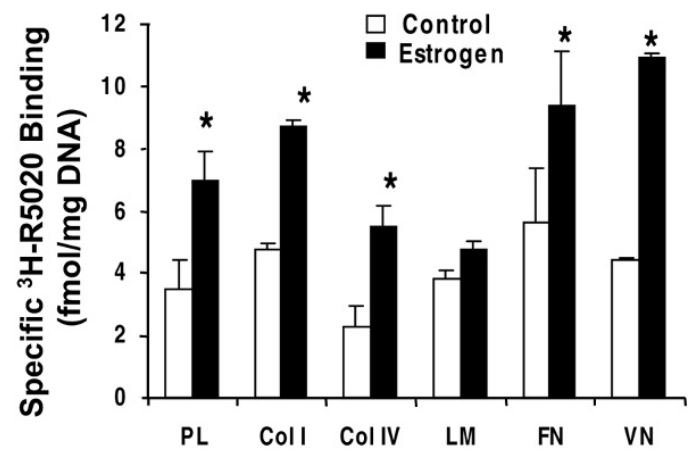

(c)

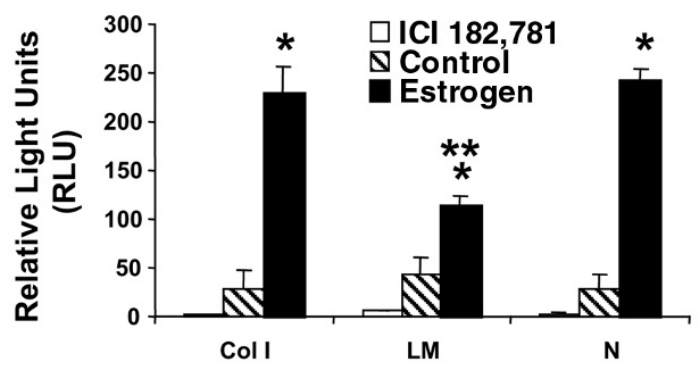

Effects of laminin on estrogen-induced proliferation, progesterone receptor regulation and estrogen receptor activity in ER positive human breast cancer cell lines. (a) MCF-7 and T47D cells were cultured in serum-free medium containing EGF ( $5 \mathrm{ng} / \mathrm{ml})$, IGF-I ( $25 \mathrm{ng} / \mathrm{ml})$ on various ECM proteins. Cells were pre-treated with $200 \mathrm{nM} \mathrm{ICl} 182,780$ for 48 hour followed by no treatment (control) or $17 \beta$-estradiol $(20 \mathrm{nM}) ;{ }^{3} \mathrm{H}$-thymidine incorporation into DNA was determined 21 hour later. ${ }^{\star} P<0.05$ that estrogen-treated groups are greater than control-treated groups. (b) MCF-7 cells were cultured in serum-free medium in the absence (control) or presence of estrogen ( $10 \mathrm{nM})$ for 3 days. Progesterone receptor

concentrations were determined by specific ${ }^{3} \mathrm{H}-\mathrm{R} 5020$ binding assay. ${ }^{*} P<0.05$ that estrogen-treated groups are greater than control groups. (c) MCF-7 cells were transfected with estrogen response element (tk109-luc) and $\beta$-galactosidase plasmids. Luciferase activity was measured 24

hour after ICl 182,780 (200 nM), control or estrogen $(10 \mathrm{nM})$ treatment. Luciferase activity was normalized to transfection efficiency determined by $\beta$-galactosidase activity/cell. ${ }^{*} P<0.05$ that estrogen-treated groups are greater than control groups. ${ }^{* *} P<0.05$ that estrogen-treated LM group is less than estrogen-treated Col I or FN groups. (Adapted from [15] with permission from Endocrinology). Col, collagen; FN, fibronectin; LN, laminin; $\mathrm{PL}$, poly-L-lysine; VN, vitronectin.

laminin, but estrogen induction of estrogen-responseelement-mediated transcription is inhibited (Fig. 5c). Tumor ECM microenvironment may thus influence epithelial cell responsiveness to hormones and may therefore lead to hormone insensitivity without the loss of hormone receptors. These studies also indicate that these ER positive cells may still be highly responsive to the mitogenic effects of growth factors and this provides a plausible explanation 
for the growth of ER positive breast cancer cells that are estrogen-independent and/or antiestrogen resistant.

We have also investigated the effects of ECM proteins on estrogen responsiveness of breast cancer cells in vivo. MCF-7 tumor cells were mixed with collagen I or laminin or both prior to implantation into athymic nude mice. Following tumor cell implantation, all mice received exogenous estrogen via estradiol-containing pellets, and tumor growth was monitored. MCF-7 cells treated with collagen I alone grew rapidly, whereas tumors cells treated with laminin exhibited dramatically reduced growth; tumors cells treated with collagen I + laminin grew at an intermediate rate. After 21 days, the estrogen pellets were removed in half the mice and these mice were then treated with the antiestrogen $\mathrm{ICl} 182,781$. Tumors arising from cells treated with collagen I alone regressed, while collagen + laminin-treated tumor growth stabilized in response to antiestrogen treatment (Haslam and Woodward, unpublished data). In contrast, the tumors arising from laminin-treated MCF-7 cells continued to grow in the presence of the antiestrogen, with tumor size increasing more than 2 -fold in 3 weeks. These results indicate that the composition of ECM that surrounds tumor cells in vivo, can affect their estrogen responsiveness and may be an important mechanism underlying antiestrogen resistance in human breast cancer.

\section{Summary and conclusion}

Breast cancer is frequently classified by ER status, since absence of hormone responsiveness is associated with a poor prognosis and substantially limits therapy options. Treatment of breast cancer with selective estrogen receptor modulators has met with limited success, partially because $40 \%$ of tumors are ER negative, $33 \%$ of ER positive tumors do not respond to endocrine therapy and all patients with advanced breast cancer on antiestrogen therapy eventually experience progression while on treatment, becoming antiestrogen resistant [18,23-26]. Our understanding, therefore, of the acquisition of hormone responsiveness in the normal breast and the loss of responsiveness in advanced breast cancer is critical to developing therapeutics to treat this disease. Acquisition and loss of estrogen action are complex events involving breast epithelial cells, their extracellular environment and signals from stromal cells. The studies we have described herein demonstrate that ECM proteins and their cellular receptors, integrins, may be critical for acquisition and loss of ovarian steroid function in normal and breast cancer cells. Furthermore, ovarian steroids regulate ECM protein and integrin expression in the mammary gland. These data indicate that the substantial changes in ECM protein expression that occur during breast cancer development and progression may directly influence tumor growth and ovarian steroid responsiveness. Advancing our understanding of the acquisition and loss of ovarian hormone status will be important in developing effective therapies for the treatment of breast cancer.

\section{Acknowledgements}

This work was supported by $\mathrm{NIH}$ grant 5R01CA40104 to $\mathrm{Dr} \mathrm{SZ}$ Haslam and DOD Grant DAMD 17-96-1-6026 to Dr TL Woodward.

\section{References}

1. Fendrick JL, Raafat AM, Haslam SZ: Mammary gland growth and development from the postnatal period to postmenopause: ovarian steroid receptor ontogeny and regulation in the mouse. J Mammary Gland Biol Neoplasia 1998, 3:7-22.

2. Bocchinfuso WP, Korach KS: Mammary gland development and tumorigenesis in estrogen receptor knockout mice. $J$ Mammary Gland Biol Neoplasia 1997, 2:323-334.

3. Cunha GR, Young P, Hom YK, Cooke PS, Taylor JA, Lubahn DB: Elucidation of a role for stromal steroid hormone receptors in mammary gland growth and development using tissue recombinant experiments. J Mammary Gland Biol Neoplasia 1997, 2:393-402.

4. Woodward TL, Xie JW, Haslam SZ: The role of mammary stroma in modulating the proliferative response to ovarian hormones in the normal mammary gland. J Mammary Gland Biol Neoplasia 1998, 3:117-131.

5. Xie J, Haslam SZ: Extracellular matrix regulates ovarian hormone-dependent proliferation of mouse mammary epithelial cells. Endocrinology 1997, 138:2466-2473.

This is the first paper to describe a role for ECM proteins in ovarian steroid action in the mammary gland.

6. Woodward TL, Xie JW, Fendrick JL, Haslam SZ: Proliferation of mouse mammary epithelial cells in vitro: interactions among epidermal growth factor, insulin-like growth factor-I, ovarian hormones and extracellular matrix proteins. Endocrinology 2000, 141:3578-3586.

This paper uses serum-free, defined culture conditions to investigate growth factor, ovarian hormone and ECM interactions involved in mammary epithelial cell proliferation.

7. Pedchenko VK, Imagawa W: Estrogen treatment in vivo increases keratinocyte growth factor expression in the mammary gland. J Endocrinol 2000, 165:39-49.

8. Hansen RK, Bissell MJ: Tissue architecture and breast cancer: the role of extracellular matrix and steroid hormones. Endocr Relat Cancer 2000, 7:95-113.

9. Sakakura T: New aspects of stroma-parenchyma relations in mammary gland differentiation. Int Rev Cytol 1991, 125:165-202.

10. Keely PJ, Wu JE, Santoro SA: The spatial and temporal expression of the $\alpha_{2} \beta_{1}$ integrin and its ligands, collagen I, collagen IV, and laminin, suggests important roles in mouse mammary morphogenesis. Differentiation 1995, 59:1-13.

This paper demonstrates that most major components of the epithelial $\mathrm{BM}$ and ECM are synthesized by stromal cells, including laminin. This in vivo study refutes previous in vitro studies that have demonstrated that laminin is synthesized by epithelial cells.

11. Simon-Assmann $P$, Simo $P$, Bouziges $F$, Haffen $K$, Kedinger $M$ : Synthesis of basement membrane proteins in the small intestine. Digestion 1990, 46:12-21.

12. Lefebvre O, Sorokin L, Kedinger M, Simon-Assmann P: Developmental expression and cellular origin of the laminin alpha2, alpha4, and alpha5 chains in the intestine. Dev Biol 1999, 210:135-150.

An innovative, but well-designed study of the cellular origin of laminin isoforms in the formation of the basement membrane in the intestine is 
presented. By using mouse/chick hybrid intestines, the authors could examine reciprocal epithelial/mesenchyme interactions in the formation of the basement membrane.

13. Haslam SZ: Mammary fibroblast influence on normal mammary epithelial cell responses to estrogen in vitro. Cancer Res 1986, 46:310-316.

14. Kenney NJ, Dickson RB: Growth factor and sex steroid interactions in breast cancer. J Mammary Gland Biol Neoplasia 1996, 1:189-198.

15. Richert MM, Wood TL: Insulin-like growth factors (IGF) and IGF type I receptor during postnatal growth of the murine mammary gland: sites of messenger ribonucleic acid expression and potential functions. Endocrinology 1999, 140:454-461.

16. Haslam SZ: Local versus systemically mediated effects of estrogen on normal mammary epithelial cell deoxyribonucleic acid synthesis. Endocrinology 1988, 122:464-470.

17. Woodward TL, Mienaltowski AS, Modi RR, Bennett JM, Haslam SZ: Fibronectin and the $\alpha_{5} \beta_{1}$ integrin are under developmental and ovarian steroid regulation in the normal mouse mammary gland. Endocrinology 2001, 142:3214-3222.

18. Paik S, Hartmann DP, Dickson RB, Lippman ME: Antiestrogen resistance in ER positive breast cancer cells. Breast Cancer Res Treat 1994, 31:301-307.

19. Ronnov-Jessen L, Petersen OW, Bissell MJ: Cellular changes involved in conversion of normal to malignant breast: importance of the stromal reaction. Physiol Rev 1996, 76:69-125.

An excellent review of the role of stromal cells and the stromal reaction

(desmoplasia) in breast cancer development and progression.

20. Rabinovitz I, Mercurio AM: The integrin alpha 6 beta 4 and the biology of carcinoma. Biochem Cell Biol 1996, 74:811-821.

21. Ziober $\mathrm{BL}$, Lin $\mathrm{CS}, \mathrm{Kramer} \mathrm{RH}$ : Laminin-binding integrins in tumor progression and metastasis. Semin Cancer Biol 1996, 7:119-128.

22. Woodward TL, Lu H, Haslam SZ: Laminin inhibits estrogen action in human breast cancer cells. Endocrinology 2000, $141: 2814-2821$

This paper describes a paracrine pathway that inhibits estrogen action. Laminin is shown to inhibit estrogen induced gene transcription. The process may have critical importance in the development of estrogen and antiestrogen resistance in breast cancer.

23. Martin MB, Saceda M, Garcia-Morales P, Gottardis MM: Regulation of estrogen receptor expression. Breast Cancer Res Trmt 1994, 31:183-189

24. Garcia M, Derocq D, Freiss G, Rochefort H: Activation of estrogen receptor transfected into a receptor-negative breast cancer cell line decreases the metastatic and invasive potential of the cells. Proc Natl Acad Sci USA 1992, 89:11538-11542.

25. Benz $\mathrm{CC}$, Brandt $\mathrm{BH}$, Zanker KS: Gene diagnostics provide new insights into breast cancer prognosis and therapy. Gene 1995, 159:3-7.

26. Osborne CK: Receptors. In Breast Diseases. Edited by Harris JR, Hellman S, Henderson LC, Kinne DW. Philadelphia: JB Lippincott Co, 1991:301-325. 